\title{
Personalized Route Planning System Based on Wardrop Equilibrium Model for Pedestrian-vehicle Mixed Evacuation in Campus
}

\author{
Pengfei Duan ${ }^{\mathrm{a}, \mathrm{b}}$, Shengwu Xiong, ${ }^{\mathrm{a}, \mathrm{b}, *}$, Chunhui Yang ${ }^{\mathrm{a}, \mathrm{c}}$, Haohao Zhang ${ }^{\mathrm{a}}$, \\ Mianfang $\mathrm{Liu}^{\mathrm{a}}$ \\ ${ }^{a}$ School of Computer Science and Technology, Wuhan University of Technology, Wuhan \\ 430070, China \\ ${ }^{b}$ Hubei Key Laboratory of Transportation Internet of Things, Wuhan University of \\ Technology, Wuhan 430070, China \\ ${ }^{c}$ Software Quality Engineering Research Center, CEPREI, Guangzhou, China
}

\begin{abstract}
Emergency evacuation is an important task while dealing with a sudden accident. Aiming at emergency evacuation in campus, this paper proposed a personalized route planning model for pedestrian-vehicle mixed evacuation. Considering the congestion of pedestrian-vehicle mixed evacuation and the actual factors of students, this model found the optimal user equilibrium close to the system equilibrium. By the help of Wardrop Equilibrium model, the server calculated the optimized evacuation route planning and guided evacuees by mobile intelligent terminals. Finally this paper applied this model to the evacuation of Wuhan University of Technology. By simulating the process of emergency evacuation, the results showed the feasibility of the algorithm, which proved this model a scientific basis for guiding the real evacuation process.

Keywords: Personalized Route Planning, Wardrop Equilibrium, Pedestrian-vehicle Mixed Evacuation
\end{abstract}

\footnotetext{
*Shengwu Xiong

Email address: swxiong@whut.edu.cn (Shengwu Xiong)
} 


\section{Introduction}

Campus security is a very important part of the social security work. A variety of dangerous factors (such as chemical experiments, fires, and other manmade disasters or accidents) have posed a threat to campus security in recent years. In the campus, buildings are complex. Students are relatively dense once a disaster occurs although they are familiar with the campus environment [1. Students tend to be nervous, loss of judgment. They are easier to follow the majority of people and choose a crowded road. It will result in missing the best evacuation time and cause a stampede, which will cause irreparable results. Thus the route planning has become one of the most important aspects in emergency evacuation to guide students to make right choices during evacuation process 2] [3].

However, most of the existing evacuation studies do not address the demands of mixed evacuation of pedestrians and vehicles. When there is an accident in campus, students will use all available transportation (such as walking, cycling and the school bus, etc.) to escape. It is possible to cause a traffic congestion and the single pedestrian evacuation model or the single vehicle evacuation model is not enough.

On the other hand, the traditional evacuation route plan is often designed with experience. It cannot change flexibly according to the actual situation and reach the best result 4 [5. With the popularity of mobile intelligent terminal and the development of mobile internet, it's feasible to apply mobile intelligent terminal to aid emergency evacuation.

In recent years, some researchers have adopted evolutionary algorithm to solve emergency evacuation problems. These models focus on the global evacuation process and finding solutions to minimize the total evacuation time or clearance time. However, these solutions may violate with some students' benefits and the students will prefer to choose the shortest route to evacuate and won't follow the route planning.

Based on the conference papers published in DCIT'15[6], this paper con- 
sidered the pedestrian-vehicle mixed evacuation model which is closer to real situation. In campus, there is no traffic light and the vehicles go through with students in the same road. The main difficulty in modeling and route planning for pedestrian-vehicle mixed evacuation lies in considering the congestion of pedestrian-vehicle mixed evacuation and the actual personalized factors of stu-

dents. For example, the crowding degree of pedestrians will affect the velocity of vehicles. In this paper, the pedestrian-vehicle mixed evacuation traffic flow model is defined. In addition, a novel personalized route planning system with Wardrop Equilibrium model is proposed to solve the mixed evacuation problem in campus.

This paper is organized as follows. Section 2 discusses previous work related to simulation and modeling for evacuation. Section 3 presents a pedestrianvehicle mixed evacuation model in campus. Section 4 introduces the campus evacuation model based on Wardrop Equilibrium model for students and makes personalized recommendation route for each one. Section 5 proposes the personalized recommendation model by using the mobile terminal. Section 6 applies the system to Nanhu campus of Wuhan University of Technology. Finally, Section 7 draws conclusions and discusses directions for future research.

\section{Related work}

For the campus emergency evacuation, $\mathrm{Chu}$ et al. [7] designed a fire escape route planning system based on mobile handheld devices and RFID technology, which can calculate the optimal escape routes according to the current positions of people while the fire beginning. Chu has brought the technology based on LBS (location based service) into the campus evacuation, but the flaw of the system was that it depended on the fire alarm system in campus, and it was difficult to guarantee the stability. Komatsu 8 proposed an automatic evacuation guiding scheme by using mobile nodes of evacuees. It automatically estimated blocked road segments from the difference between the presented evacuation route and the actual evacuation route, and then recalculated the alternative 
evacuation route. But it is important to consider the evacuee's actual factors such as the personal physique. By analyzing the evacuation process in large building, Sun 9 summarized the characteristics of emergency evacuation, and proposed the fire evacuation scheme for teaching building. She got the personnel evacuation time and found the bottleneck phenomenon of evacuation process by simulation experiment. Sun's study has established the simulation model, but it lacked in the simulation of microscopic behavior such as the impact of obstacle. There are also many other researches [10, 11, 12, 13, 14, of studying various methods to optimize the routes in the evacuation process.

Current studies on mixed traffic flow usually focus on simulation[15]. Xie et al.[16] proposed a new two-dimensional car-following model to depict the features of mixed traffic flow consisting of motorized vehicles and non-motorized vehicles based on the two-dimensional optimal velocity model. In the model, velocity difference terms are introduced, which are regarded as important factors for traffic behavior. Numerical simulations are carried out to investigate the interaction between left-turning vehicle flow and straight-going vehicle flow at a typical unsignalized interaction. Almodfer et al. [17 proposed a lane-based approach for evaluating the post-encroachment time between pedestrians and vehicles. Almodfer analyzed the lane-based distribution of pedestrian-vehicle conflict by using collected video data which recorded the behaviors of vehicles and pedestrians in a non-signalized marked crosswalk in Wuhan, China, and discussed the effect of waiting time on lane-based pedestrian-vehicle conflict as well as the distribution of pedestrian walking speed under different levels of severity of lane-based pedestrian-vehicle conflict. These studies mainly focused on the microscopic evacuation behavior of pedestrians or vehicles. But the real evacuation scenarios need to find the optimal route planning considering to reduce the congestion of pedestrian-vehicle mixed travel.

Currently, many optimization algorithms have been used to simulate and solve evacuation problems for making evacuation plans. Duan and Xiong [18] proposed an ant colony optimization model based on guidance degree. The guidance degree is proposed to combine factors which influence students' deci- 
sions during evacuation. However, students will prefer to choose the shortest route to exits while being evacuated and won't follow the route planning. Considering the actual reaction of students, the purpose of our work is to propose a personalized route planing system based on Wardrop Equilibrium model to find a feasible route planning.

\section{Pedestrian-vehicle Mixed Evacuation Model in Campus}

\subsection{Campus evacuation network description}

In the campus enviroment, the building layout is more complex than the outside. When there is an accident, students will use all the possible way to escape. such as playground, square, etc. So the vacancy area should be considered for emergency evacuation. As it is shown in Fig. 1, these vacancy areas

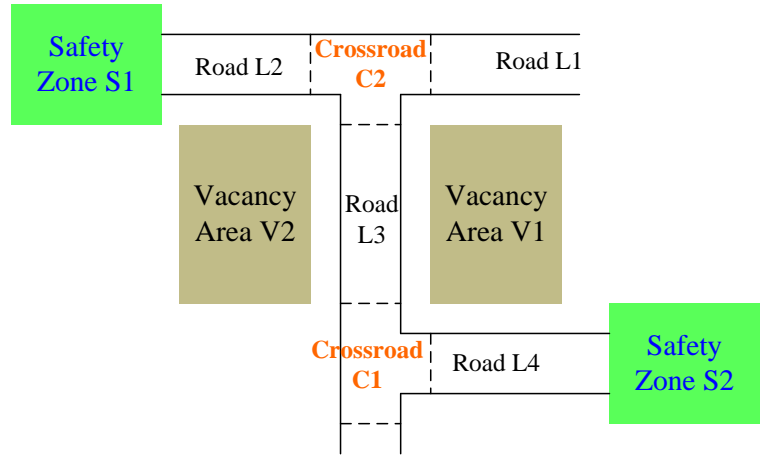

Figure 1: Distribution of campus road

can be treated as evacuation nodes and they are connected to other neighboring nodes to express the connectivity. By this way, The evacuation network is set up as Fig. 2.

The campus evacuation network can be represented as $G(V, E)$, where $V$ is the set of nodes and $E$ is the set of roads. It shows as follow:

$$
\begin{gathered}
V=<v_{1}, v_{2}, \ldots, v_{n}> \\
E \subseteq V * V
\end{gathered}
$$




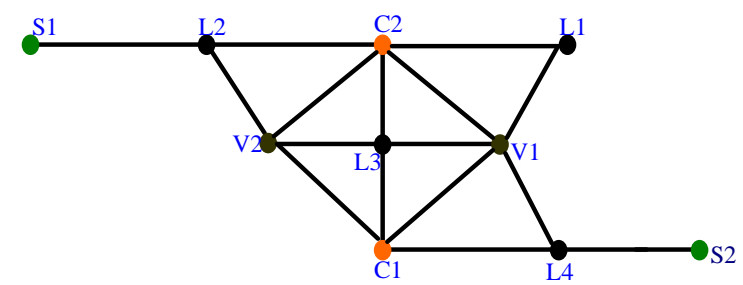

Figure 2: Extraction of campus road network

The properties of node $_{i}$ include flow capacity, position, maximum traffic capacity, etc.

\subsection{Pedestrian-vehicle mixed evacuation problem}

Pedestrian-vehicle mixed evacuation refers to solve the problem that many pedestrians together with vehicles need to be transferred out of an integrated environment with buildings and surrounding roads in an emergency situation.

During the evacuation process, the traffic congestion is one of the most crucial issues in multi-mode transportation. A large number of pedestrians and vehicles will put great pressure on road network. Once there is a congestion, the whole evacuation time will be greatly extended and the efficiency of the evacuation will be reduced sharply.

Every road $\operatorname{road}_{i}$ has a saturation value which is defined as $F_{i} / C_{i} . F_{i}$ is the traffic flow of road $i$ and $C_{i}$ is the capacity.

To study the saturation value of pedestrian-vehicle mixed traffic, we used crowding degree which represents the traffic congestion of evacuees at time $t$. It shows as the following formula:

$$
w_{i}=e^{r F_{i} / C_{i}-0.5}
$$

Where $C_{i}$ can be represented by the size of road $i$ and $r$ is the parameter for different type of road. For example, a weak road will cause the congestion easily and the bigger $r$ is needed.

For pedestrian-vehicle mixed traffic, the different sizes of evacuees have different effects on the crowding degree, which is shown as Fig. 3. To get the 


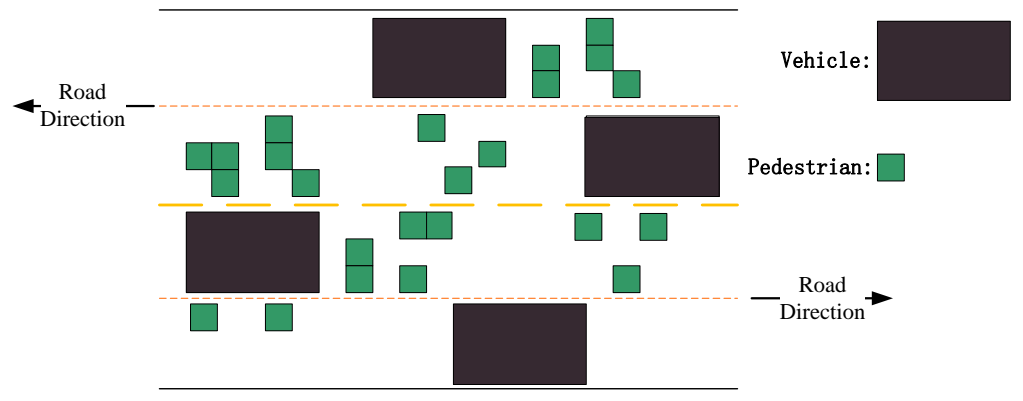

Figure 3: The multi-source and multi-destination network

crowding degree of the mixed-traffic road, we convert them to a unified standard which is shown as follows:

$$
\bar{F}_{i}=N_{c a r} * S_{c a r}+N_{p e p} * S_{p e p}
$$

where $F_{i}$ is the total traffic flow for pedestrian-vehicle mixed traffic, $N_{c a r}$ is the number of vehicles in a special road and $S_{c a r}$ is the size occupied by a vehicle. $N_{p e p}$ is the number of pedestrians and $S_{p e p}$ is the size of one pedestrian.

In a congestion situation, the evacuation speed is affected by the saturation value. We defined the relation as the following formula:

$$
v= \begin{cases}V_{\max }, & \text { if } r F_{i} / C_{i}<0.5 \\ V_{\max } * \frac{1}{e^{r F_{i} / C_{i}-0.5}}, & \text { Otherwise }\end{cases}
$$

Where $V_{\max }$ is the max velocity of free traffic flow. When the crowding degree is greater than 0.5 , pedestrians or vehicles are congested with each other and their max velocity will be affected.

\section{Route Planning for Campus Evacuation based on Wardrop Equi- librium Model}

The underlined problem of campus emergency evacuation is multi-source and multi-destination. In campus evacuation, the spatial topology of the campus can be represented as a network $G(V, E)$, where $V$ represents a set of vertices, $E$ is the set of edges with weights. As it's shown in Fig. 4. During the evacuation, 
students and vehicles start from different locations and finally evacuate from different outlets.

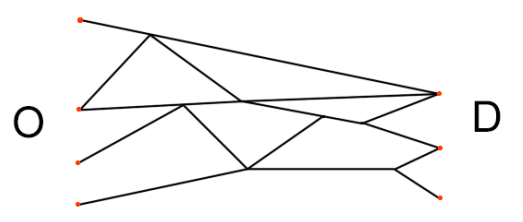

Figure 4: The multi-source and multi-destination network

In emergency evacuation, everyone tends to find the shortest route to exits under the user equilibrium, but it will cost more time than system equilibrium. So it is necessary to find the user equilibrium which is closer to system equilibrium.

\subsection{Wardrop Equilibrium Based on Genetic Algorithm}

Wardrop Equilibrium is commonly used as a solution concept of network games such as modeling transportation and telecommunication networks with congestion. It was proposed by John Glen Wardrop in 1952 [19] and became a mathematical formalization by Beckmann [20].

The simplified transport network of Wardrop Equilibrium is expressed as the Fig. 5. Because of congestion, the time or cost from $O$ to $D$ is changing between

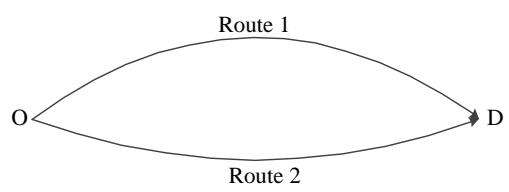

Figure 5: Simplified transport network

route 1 and route 2 . Each student usually choose a route that minimizes the time or cost incurred in its traversal. It can be seen that there is a balance point for the two routes and it can be called user equilibrium. 


\subsection{Definition of Variables and Parameters}

The emergency evacuation path network can be defined as a directed graph, $G=(V, E)$. There is a set of OD pairs, $K \subseteq V \times V$. Let $P_{k}$ be the set of directed paths in $G$ from $s_{k}$ to $t_{k}$.

$h_{k}^{r s}$ : The traffic flow in link $r s$ for route $k$.

$c_{k}^{r s}$ : The traffic cost in link $r s$ for route $k$.

$c^{r s}$ : The traffic cost in the shortest link $r s$.

$t^{r s}$ : The traffic time in the shortest link $r s$.

This paper adopts BPR(Bureau of Public Road) as the link performance functions [21:

$$
T_{a}\left(x_{a}, C_{a}\right)=t_{a}^{0}\left[\left(1+\alpha\left(\frac{x_{a}}{C_{a}}\right)^{\beta}\right], \forall a \in E\right.
$$

Where $t_{a}^{0}$ is the free cost time, $C_{a}$ is the capacity of link and $T_{a}$ is the travel time with the flow $x_{a} . \alpha$ and $\beta$ are the parameters of BPR.

\subsection{Model Formulation}

In this model, we will convert student and vehicle to a unified unit $u$, and units which stand for vehicle will move together in the simulation. Each unit can't get out of campus with shorter time under user equilibrium, but local optimal user equilibrium usually exists. It is valuable to find the user equilibrium close to the system equilibrium. This paper proposed the Wardrop Equilibrium model based on genetic algorithm.

The model of Wardrop Equilibrium can be expressed as follows:

$$
\min Z(x)=\sum_{a \in A} x_{a} c_{a}\left(x_{a}\right)
$$

Subject to the following constraints:

$$
\begin{gathered}
\sum_{k} h_{k}^{r s}=t^{r s}, \forall r s \in \Omega \\
x_{a}=\sum_{k} \sum_{r s \in \Omega} \delta_{a, k}^{r s} f_{k}^{r s}, \forall a \in E \\
h_{k}^{r s} \leq 0, x_{a} \leq 0
\end{gathered}
$$


Where $k=\left(s_{k}, t_{k}\right) \subseteq K$ and $\delta_{a, k}^{r s} \in\{0,1\}$ shows that if the route $k$ include the arc $a$ in link $r s$.

Considering the reaction of pedestrian and vehicle in emergency evacuation, the ideas of Wardrop equilibrium model based on genetic algorithm in campus evacuation are reviewed as follows:

View $N$ as the number of units to be evacuated and $M$ as the population of Wardrop equilibrium. Take into account $Z(x)$ of system equilibrium as the fitness of genetic algorithm. Adopt the crossover and mutation as the process of finding the best user equilibrium. The process of the model can be shown as follow:

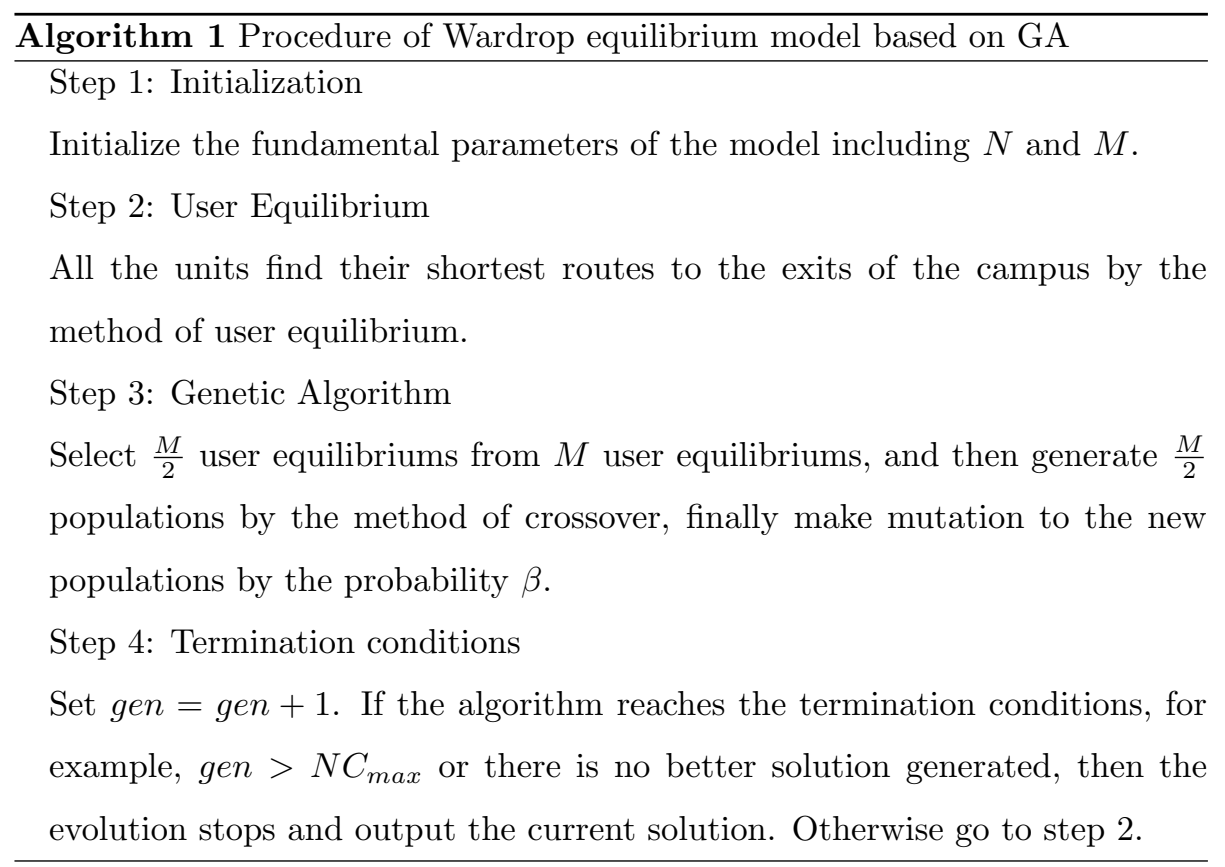

\section{Personalized Route Planning System based on Wardrop Equilib- rium}

In this section, we described the components of the personalized route planning system, and introduced the way to combine these parts and wardrop equi- 
librium model to be a whole system to solve the problem of emergency evacuation in campus.

\subsection{System Design}

There are four main models in this system: map, evacuation, service and personal settings. The functions of the system are:

1) Registration and collecting the students' information;

2) Terminal location statistics which provides the distributed location information for emergency evacuation of school;

3) Personalized route planning in emergency evacuation.

\subsection{Personalized Route Generation}

When a disaster occurs, students tend to select a different destination according to their different preference. There are so many factors that affect the route choice, such as the dormitories, transportation methods, the distribution of evacuees and personal physique.

The user can input the information such as the student's name, student ID and transportation methods in personal settings page. It is shown in Fig. 6. Through the student ID, students' dormitories in campus can be associated, which effect personalized routes planning in the process of evacuation. If the end of the evacuation route distance is far away from the students' dormitory, the fitness will be reduced, so as to achieve the evacuation path of personalized design.

The system consists of a server and clients. As the client, smartphones will send their positions to the server. The server will use the data received from clients to implement route planning, then return the route to the client. The main framework is shown in Fig. 7 .

\section{Experiment}

This paper applied the system to the Nanhu campus of Wuhan University of Technology to validate the practicability of this model. The satellite image 


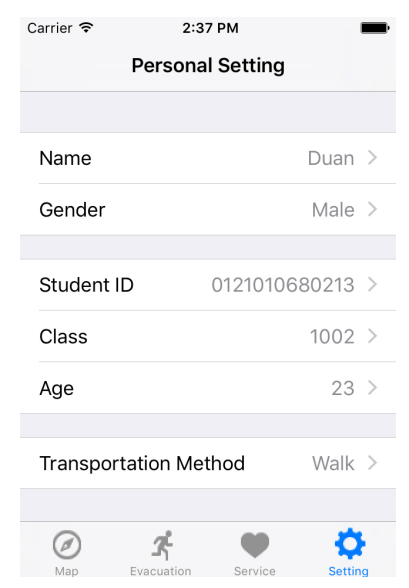

Figure 6: Personal settings of system

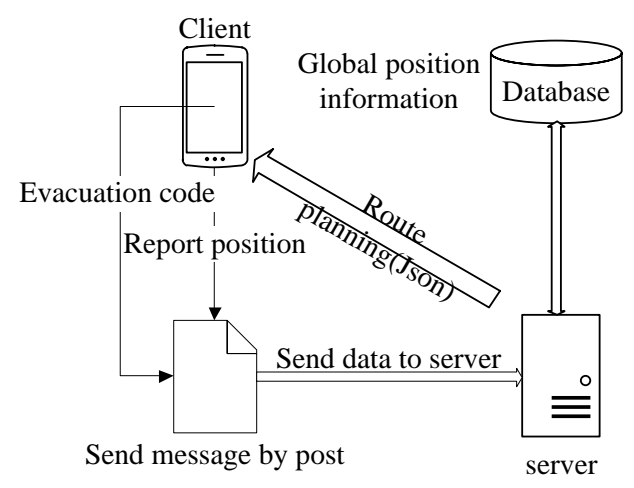

Figure 7: System framework

of the campus is shown in Fig. 8.

In this campus, there are three teaching buildings, one parking area and a group of dormitories. To simulate the actual mixed-traffic situation, we arranged 15000 students and 200 cars to be distributed in the campus. According to the students' course timetable, students were arranged in the teaching buildings and dormitories. The 200 cars were in the parking area. The detailed setting of the experiment shows in Table 1.

By analysis of the road network structure of campus, There are 63 nodes of road and four exits in the campus. It shows in Fig. 9. 


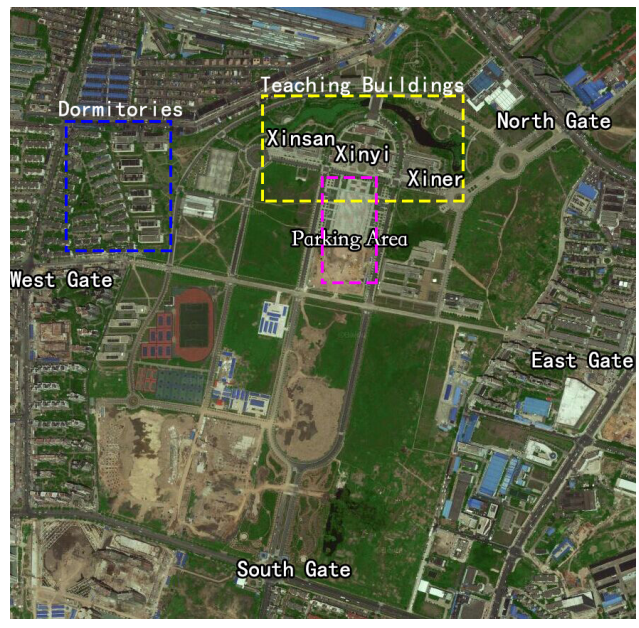

Figure 8: The satellite image of Nanhu campus in Wuhan University of Technology

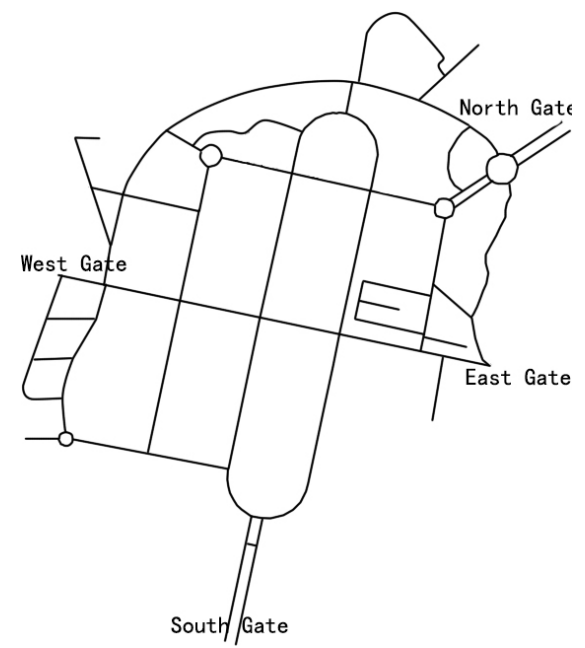

Figure 9: Road network of Nanhu campus in Wuhan University of Technology

After the initialization of evacuees' locations, the system began to calculate the route planning according to the Wardrop Equilibrium model and the preference of the evacuees. The evacuation procedure was shown in Fig. 10. In the experiment, 15000 students and 200 cars needed to be evacuated and the routes were drawn on the picture with different colors and width. 
Table 1: Experiment parameter setting

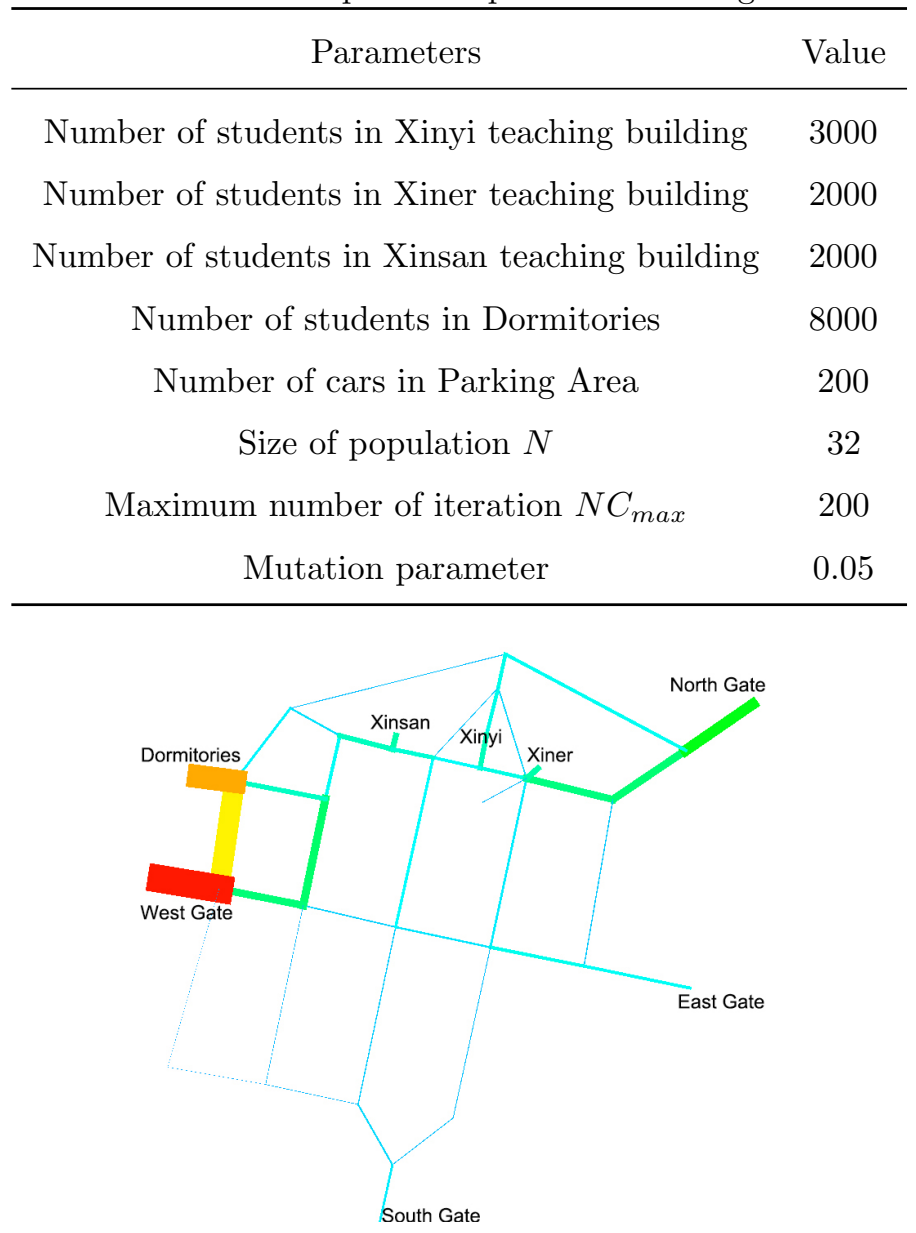

Figure 10: Simulation Results

In order to analyze the advantage of our method, we made a comparison with the evacuation clearance time among the proposed evolutionary wardrop equilibrium model, K-short model, user equilibrium model(that means everyone choose shortest path) and system equilibrium model(implemented by ACO method and focus on the shortest clearance time). The spatial-temporal analysis is shown in Fig. 11. We can see that K-short model arranges most of the evacuees' routes through the West door and North door which will lead to con- 
gestion. The other three models make the most of the East door and the North door which will reduce the clearance time.

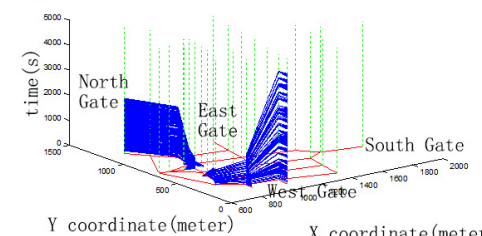

$$
\text { (a) } \mathrm{K} \text {-short model }
$$

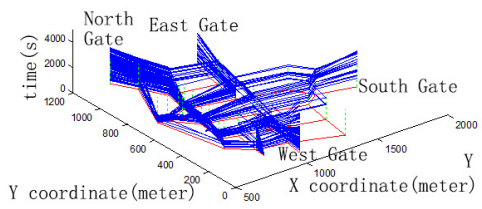

(c) User equilibrium model

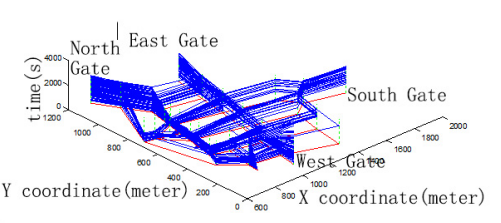

(b) System equilibrium model

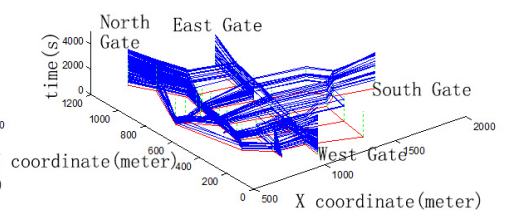

(d) Evolutionary wardrop equilibrium model

Figure 11: Spatial-temporal analysis of emergency evacuation

The final evacuation procedures and clearance time are shown in Fig. 12 and Table 2. We can see that the K-short model's evacuation clearance time is longest(4431s). Our model provides a better solution(2301s) than user equilibrium model(2388s), a little longer than system equilibrium model(2297s). But the system equilibrium model doesn't take every evacuee's benefit into consideration and makes a longer route planning for someone. It is hard to carry out the plan because it violates someone's benefit.

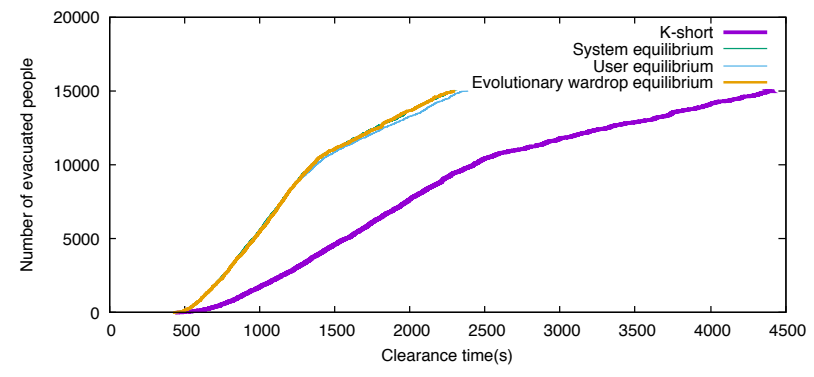

Figure 12: Evacuation clearance time comparison chart

Considering every students' requirements, the system recommended person- 
Table 2: Comparison of the clearance time among different models

\begin{tabular}{ccc}
\hline Model & Experiment No. & Clearance time(s) \\
\hline K-short & 1 & 4431 \\
\hline \multirow{2}{*}{ System equilibrium } & 1 & 2297 \\
& 2 & 2293 \\
& 3 & 2290 \\
\hline User equilibrium & 1 & 2388 \\
& 2 & 2401 \\
Evolutionary wardrop equilibrium & 2 & 2393 \\
\hline & 3 & 2301 \\
& 3 & 2313 \\
& 2328 \\
\hline
\end{tabular}

alized routes for different students by combining their different preferences. As it was show in Fig. 13, student A and student B were arranged different routes by their dormitories.
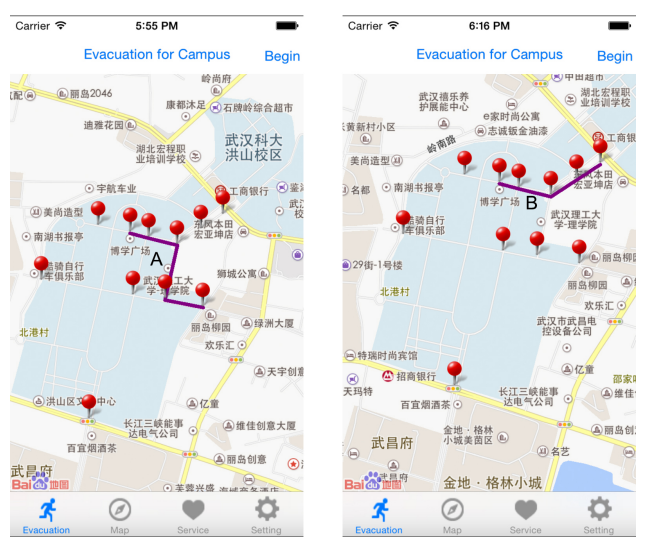

Figure 13: Routes for student A and student B 


\section{Conclusion}

Combining with the intelligent terminal, this paper proposed an emergency evacuation system based on Wardrop Equilibrium model. It made good use of the advantage of mobile terminals which are popularized in student groups and the route searching ability of Wardrop Equilibrium for campus evacuation. Considering the different factors of students while choosing path in the process of emergency evacuation, this system shows the scientificity and feasibility .

\section{Acknowledgment}

This work was supported in part by the National High-tech R\&D Program of China (863 Program) under Grant No.2015AA015403, National Science Foundation of China under Grant No.61170202, Hubei Key Laboratory of Transportation Internet of Things under Grant No. 2015III015-B03, Science \& Technology Pillar Program of Hubei Province under Grant No.2014BAA146 and Nature Science Foundation of Hubei Province under Grant No.2015CFA059.

\section{References}

[1] K. Bianmin, W. Wei, Z. Ruifeng, Modeling and evaluating emergency evacuation strategy for student residential hall, Mathematics in Practice and Theory, 2014, 44(23): 1-6.

[2] Y. Inoue, T. Ikeda, K. Yamamoto, T. Yamashita, A. Sashima and K. Kurumatani, Usability Study of Indoor Mobile Navigation System in Commercial Facilities, Ubiquitous Systems Evaluation 2008 (USE '08) in Ubi-Comp 2008, Seoul, Korea (2008).

[3] A. Ahmed and N. Fisal, A real-time routing protocol with load distribution in wireless sensor networks, Computer Communications, 2008, 31(14): 31903203. 
[4] L. Jingling, J. Qingge, Research on GA-based optimizing multi-exit evacuation path problem, Journal of Shanghai Normal University(Natural Sciences), 2008, 37(4): 383-389.

[5] D. Pengfei, Research on Equilibrium Model and Optimization Medthod for Campus Evacuation, Wuhan university of technology, 2013.

[6] D. Pengfei, Z. Haohao, X. Shengwu, Z. Siqin, C. Zhong, Y. Pengcheng, Personalized Route Planning System Based on Wardrop Equilibrium Model for Campus Evacuation, The Second International Symposium on Dependable Computing and Internet of Things, Wuhan, 2015.

[7] L. Chu, A RFID-based hybrid building fire evacuation system on mobile phone, New Jersey: Proceedings 2010 6th International Conference on Intelligent Information Hiding and Multimedia Signal Processing, IIHMSP, 2010.

[8] N. Komatsu, M. Sasabe, J. Kawahara, et al, Automatic Evacuation Guiding Scheme Using Trajectories of Mobile Nodes, Mobile Web and Intelligent Information Systems. Springer International Publishing, 2015: 3-14.

[9] S. Li, T. Jianhui, P. Taoying, Research of the evacuation number and speed in campus teaching building, Intelligence, 2008, 19.

[10] J. Izquierdo, I. Montalvo, R. Prez, and V. S, Fuertes, Forecasting pedestrian evacuation times by using swarm intelligence, Physica A, 2009, 388: 12131220.

[11] F. Tingyong, Y. Jufen, W. Xiaokun, Simulation study on occupant evacuation characteristics under emergent conditions, Journal of Applied Fire Science, 2010, 20(1): 21-38.

[12] L. Shan, L. Wenshuo, W. Xun, L. Fangzhou, Study on evacuation strategy in high-rise buildings fire, Advanced Manufacturing Technology, 2012, 472: 3207-3213. 
[13] L. Chong, Q. Changhua, Simplified evacuation planning model on naval ship, Applied Mechanics and Materials, 2011, 44: 1739-1744.

[14] Y. Liu, S. Xiong, A Fine-grained Parallel Multi-Objective Genetic Algorithm for Stadium Evacuation Route Assignment, International Journal of Digital Content Technology and its Applications, 2012, 6(8):302-310.

[15] Q. Li, B. Wang Properties of vehicle gap distribution in the mixed traffic flow. Proc Eng, 2012, 31:1001-5.

[16] D. Xie, Z. Gao, X. Zhao, et al. Characteristics of mixed traffic flow with non-motorized vehicles and motorized vehicles at an unsignalized intersection. Physica A, 2009, 388(10): 2041-2050.

[17] R. Almodfer, S. Xiong, Z. Fang Z, et al. Quantitative analysis of lane-based pedestrian-vehicle conflict at a non-signalized marked crosswalk. Transportation Research Part F: Traffic Psychology and Behaviour, 2015.

[18] D. Pengfei, X. Shengwu, J. Hongxin, Ant Colony Optimization based on Guidance Degree for Emergency Evacuation, International Journal of Digital Content Technology and its Applications, 2012, 6(20): 607-615.

[19] J. G. Wardrop, J. I. Whitehead, Correspondence. Some Theoretical Aspects of Road Traffic Research, ICE Proceedings: Engineering Divisions, 1952, 1(5): 767 .

[20] M. J. Beckmann, C. B. McGuire, C. B. Winsten, Studies in the Economics of Transportation, Yale University Press, New Haven, CT, 1956.

[21] W. Zengxin, S. Ting, G. sujian, Stochastic User Equilibrium Model and Algorithm for Elastic Demand Level Road Network, Statistics and Decision, 2012, 7: 79-81. 